\title{
Data report: characteristics of sedimentary phosphorus at North Pond, IODP Expedition 336'
}

\author{
Delphine Defforey ${ }^{2}$ and Adina Paytan ${ }^{2,3}$
}

\section{Chapter contents}

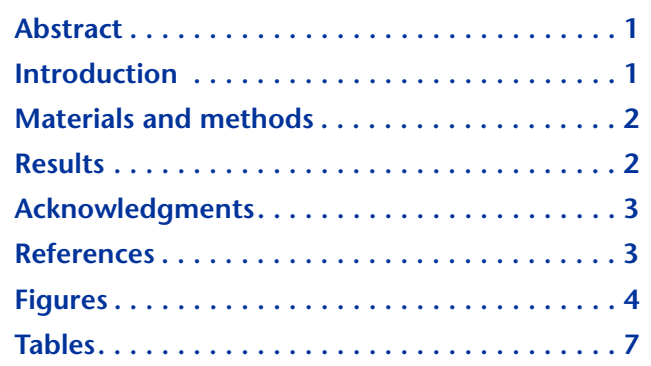

${ }^{1}$ Defforey, D., and Paytan, A., 2015. Data report: characteristics of sedimentary phosphorus at North Pond, IODP Expedition 336. In Edwards, K.J., Bach, W., Klaus, A., and the Expedition 336 Scientists, Proc. IODP, 336: Tokyo (Integrated Ocean Drilling Program Management International, Inc.). doi:10.2204/iodp.proc.336.205.2015 ${ }^{2}$ Department of Earth and Planetary Sciences, University of California, Santa Cruz, 1156 High Street, Santa Cruz CA 95064, USA. Correspondence author: ddeffore@ucsc.edu ${ }^{3}$ Also at: Institute of Marine Sciences, University of California, Santa Cruz, 1156 High Street, Santa Cruz CA 95064, USA.

\begin{abstract}
Phosphorus (P) plays an important role in fueling life, including microbial life in the deep subseafloor environment, which is estimated to contain up to 1\% of Earth's total biomass. These microorganisms play a significant role in controlling the chemical composition of the deep ocean and atmosphere on geological timescales by selectively degrading organic matter through metabolic respiration. Consequently, understanding $\mathrm{P}$ geochemistry in subseafloor sediments is important, as P bioavailability can impact microbial activity. This study focuses on characterizing and quantifying the main reservoirs of solid-phase $\mathrm{P}$ in open-ocean sediments. The sediment samples used in this study were collected during Integrated Ocean Drilling Program (IODP) Expedition 336 to North Pond, a sediment pond located on the western flank of the Mid-Atlantic Ridge. We characterized solid-phase P reservoirs in sediments from four holes (U1384A, U1382B, U1383D, and U1383E) using the sedimentary extraction (SEDEX) sequential extraction scheme. This method quantitatively separates five distinct sedimentary $P$ reservoirs: (1) loosely sorbed $P$, (2) ferric iron-bound $\mathrm{P},(3)$ authigenic carbonate fluorapatite + biogenic apatite $+\mathrm{CaCO}_{3}$-associated $\mathrm{P}$, (4) detrital apatite, and (5) refractory organic P. The separation of these P-bearing phases is based on the reactivity of each targeted phase to a particular extractant solution.
\end{abstract}

\section{Introduction}

Phosphorus (P) is an essential nutrient for life and is present in a wide range of organic and inorganic compounds, which are characterized by different bioavailability and reactivity (Björkman and Karl, 1994; Paytan and McLaughlin, 2007). Elucidating P geochemistry in open-ocean sediments is important, considering little is known about the nature of $\mathrm{P}$ compounds fueling microbial life in the deep subseafloor environment. This environment is estimated to contain up to 1\% of Earth's total biomass and includes significant prokaryotic populations at depths greater than $1 \mathrm{~km}$ (Kallmeyer et al., 2012; Roussel et al., 2008). The existence and activity of these microbial populations may have implications on global biogeochemical cycles and our understanding of the limits of life. This study aims to characterize the solid-phase P reservoirs in sediment samples collected from North Pond, a sediment pond 
on the western flank of the Mid-Atlantic Ridge sampled during Integrated Ocean Drilling Program (IODP) Expedition 336 (Fig. F1), using the sedimentary extraction (SEDEX) sequential extraction scheme (Ruttenberg, 1992; Ruttenberg et al., 2009). The appeal of the SEDEX method is that it has been successfully used in numerous studies in the field of P biogeochemistry (Ruttenberg, 2003). Furthermore, it is the only procedure that allows for the separation of carbonate fluorapatite from the detrital apatite pool (Ruttenberg, 1992). This is critical because authigenic apatite represents a sink for reactive $\mathrm{P}$, whereas detrital apatite does not (Delaney, 1998).

\section{Materials and methods}

Solid-phase $\mathrm{P}$ concentrations in different reservoirs are determined following the SEDEX sequential extraction procedure. This method separates sedimentary $\mathrm{P}$ into five operationally defined categories: (1) loosely sorbed P, (2) ferric iron-bound P (abbreviated Fe-bound), (3) authigenic carbonate fluorapatite + biogenic apatite $+\mathrm{CaCO}_{3}$-associated $\mathrm{P}$ (abbreviated auth/bio apatite $+\mathrm{CaCO}_{3}$-bound $\left.\mathrm{P}\right)$, (4) detrital apatite, and (5) refractory organic P (Ruttenberg et al., 2009). The separation of sedimentary $P$ pools is based on the reactivity of each given pool to a particular extractant solution. Briefly, frozen squeezed sediment cakes are lyophilized, ground, and sieved to $<125 \mu \mathrm{m}$. Weighed samples $(0.08 \mathrm{~g})$ are extracted along with an internal standard for reproducibility. The procedure begins with a $1 \mathrm{M}$ magnesium chloride $\left(\mathrm{MgCl}_{2}\right)$ extraction that targets phosphate loosely bound to mineral phases. The extraction is followed by a reductive step during which sodium dithionite reduces iron oxyhydroxides and citrate complexes with iron, thus releasing $\mathrm{P}$ associated with iron oxides. The residue is then extracted in 1 $\mathrm{M} \mathrm{MgCl}_{2}$ to remove any residual oxide-bound $\mathrm{P}$. Next, authigenic/biogenic apatite and $\mathrm{P}$ bound to calcium carbonate are extracted in $1 \mathrm{M}$ sodium acetate, and any residual $\mathrm{P}$ from that pool is extracted in two consecutive $1 \mathrm{M} \mathrm{MgCl}_{2}$ extractions. Following this step, detrital apatite is extracted in $1 \mathrm{M}$ hydrochloric acid $(\mathrm{HCl})$. Lastly, refractory organic $\mathrm{P}$ and residual $\mathrm{P}$ are extracted in $1 \mathrm{M} \mathrm{HCl}$ following ashing of sample residues at $550^{\circ} \mathrm{C}$. Total $\mathrm{P}$ concentrations in sediment extracts are measured using inductively coupled plasma-optical emission spectroscopy (ICPOES) on axial mode (Perkin-Elmer Optima 4300 DV Inductively Coupled Plasma Optical Emission Spectrometer operated by the University of California, Santa Cruz). Standards are prepared with the same solutions as those used for the SEDEX procedure in order to minimize matrix effects on P measurements. Sediment extracts and standards $(0,0.1,1$, and 10 $\mathrm{ppm}$ ) are diluted to lower salt content to prevent salt buildup on the nebulizer. Concentration data from both wavelengths (213 and $214 \mathrm{~nm}$ ) are averaged to obtain extract concentrations for each sample. The detection limit for $\mathrm{P}$ on this instrument for both wavelengths is $11 \mathrm{ppb}$. Low-P blanks associated with polycarbonate filters in the manifold reaction vessels were determined for each extraction step and subtracted from sample $\mathrm{P}$ measurements. We determined the precision for each SEDEX extraction step using repeat extraction of an internal sediment standard and analyses by inductively coupled plasma optical emission spectroscopy to be $28.4 \%, 8.7 \%$, $11.4 \%, 17.0 \%$, and $15.4 \%$ for steps $1-5$, respectively. The higher error associated with loosely sorbed $\mathrm{P}$ measurements is due to the low P concentrations in those sediment extracts, which introduces uncertainty when measuring P concentrations on the ICPOES.

\section{Results}

Solid-phase $\mathrm{P}$ at North Pond is mainly present in mineral phases. The most abundant geochemical forms of sedimentary P are (1) P adsorbed onto iron oxyhydroxides and (2) $\mathrm{P}$ present in authigenic/biogenic apatite and $\mathrm{P}$ bound to calcium carbonate (Figs. F2, F3; Tables T1, T2, T3). Organic P is also present in small amounts throughout the sediment core, even at deep sections of the sedimentary column (Figs. F2, F3; Tables T1, T2, T3). These results suggest that $\mathrm{P}$ bioavailability to the deep biosphere is highly impeded since mineral forms are considered to be less labile for microorganisms. However, buried organic P could constitute a bioavailable P source to the deep biosphere. Alternatively, it is possible that deep subseafloor microorganisms have mechanisms to utilize mineral $\mathrm{P}$.

Total solid-phase $\mathrm{P}$ concentrations measured fall within the ranges of concentrations measured for other open-ocean regions (Filippelli, 1997). Total sedimentary $\mathrm{P}$ content varies little throughout the sediment column, with the exception of sediment layers near the sediment/basement interface. Solidphase $\mathrm{P}$ content is greatest at 91.9 meters below seafloor (mbsf) in Hole U1384A and at $89.1 \mathrm{mbsf}$ in Hole U1382B (Fig. F2). To a lesser extent, a similar increase in $\mathrm{P}$ is observed in Hole U1383E at 42.7 mbsf but is absent in Hole U1383D (Fig. F3). The increase in $\mathrm{P}$ content downcore could be indicative of higher $\mathrm{P}$ accumulation rates when those sediment layers were deposited. 


\section{Acknowledgments}

We thank IODP Expedition 336 science party members and IODP for collecting and providing us with sediment samples for this study. Funding was provided by a graduate fellowship from the Center for Dark Energy Biosphere Investigations (NSF C-DEBI) to D. Defforey (award \# 157598) and a research grant from C-DEBI to A. Paytan (award \# 156246). We also thank Rob Franks, Michael Kong, Caitriona Berger, and Natalie Zimdahl for their assistance with this project. This is C-DEBI contribution 256.

\section{References}

Björkman, K., and Karl, D.M., 1994. Bioavailability of inorganic and organic phosphorus compounds to natural assemblages of microorganisms in Hawaiian coastal waters. Marine Ecology Progress Series, 111(3):265-273. http://dx.doi.org/10.3354/meps111265

Delaney, M.L., 1998. Phosphorus accumulation in marine sediments and the oceanic phosphorus cycle. Global Biogeochemical Cycles, 12(4):563-572. http:// dx.doi.org/10.1029/98GB02263

Expedition 336 Scientists, 2012. Sediment and basement contact coring. In Edwards, K.J., Bach, W., Klaus, A., and the Expedition 336 Scientists, Proceedings of the Integrated Ocean Drilling Program, 336: Tokyo (Integrated Ocean Drilling Program Management International, Inc.). http://dx.doi.org/10.2204/

iodp.proc.336.106.2012

Filippelli, G.M., 1997. Controls on phosphorus concentration and accumulation in oceanic sediments. Marine Geology, 139(1):231-240. http://dx.doi.org/10.1016/ S0025-3227(96)00113-2

Kallmeyer, J., Pockalny, R., Adhikari, R.R., Smith, D.C., and D'Hondt, S., 2012. Global distribution of microbial abundance and biomass in subseafloor sediment. Pro- ceedings of the National Academy of Sciences of the United States of America, 109(40):16213-16216. http:// dx.doi.org/10.1073/pnas.1203849109

Paytan, A., and McLaughlin, K., 2007. The oceanic phosphorus cycle. Chemical Reviews, 107(2):563-576. http:// dx.doi.org/10.1021/cr0503613

Roussel, E.G., Cambon Bonavita, M.-A., Querellou, J., Cragg, B.A., Webster, G., Prieur, D., and Parkes, R.J., 2008. Extending the sub-sea-floor biosphere. Science, 320(5879):1046. http://dx.doi.org/10.1126/science. 1154545

Ruttenberg, K.C., 1992. Development of a sequential extraction method for different forms of phosphorus in marine sediments. Limnology and Oceanography, 37(7):1460-1482. http://dx.doi.org/10.4319/ 10.1992.37.7.1460

Ruttenberg, K.C., 2003. The global phosphorus cycle. In Holland, H.D., and Turekian, K.K. (Eds.), Treatise on Geochemistry (Vol. 8): Biogeochemistry: New York (Elsevier), 585-643. http://dx.doi.org/10.1016/B0-08-043751-6/ 08153-6

Ruttenberg, K.C., Ogawa, N.O., Tamburini, F., Briggs, R.A., Colasacco, N.D., and Joyce, E., 2009. Improved, highthroughput approach for phosphorus speciation in natural sediments via the SEDEX sequential extraction method. Limnology and Oceanography: Methods, 7(5):319-333. http://dx.doi.org/10.4319/ lom.2009.7.319

Ryan, W.B.F., Carbotte, S.M., Coplan, J.O., O’Hara, S., Melkonian, A., Arko, R., Weissel, R.A., Ferrini, V., Goodwillie, A., Nitsche, F., Bonczkowski, J., and Zemsky, R., 2009. Global multi-resolution topography synthesis. Geochemistry, Geophysics, Geosystems, 10(3):Q03014. http://dx.doi.org/10.1029/2008GC002332

Initial receipt: 15 October 2014

Acceptance: 4 June 2015

Publication: 2 September 2015

MS 336-205 
Figure F1. Bathymetric map of North Pond showing the locations of the sites sampled during Expedition 336 (white dots). Contour lines $=100 \mathrm{~m}$ intervals. Inset $=$ location of North Pond field site on the western flank of the Mid-Atlantic Ridge. This map was created using the default Global Multi-Resolution Topography Synthesis basemap (Ryan et al., 2009) in GeoMapApp version 3.4.1 (http://www.geomapapp.org).

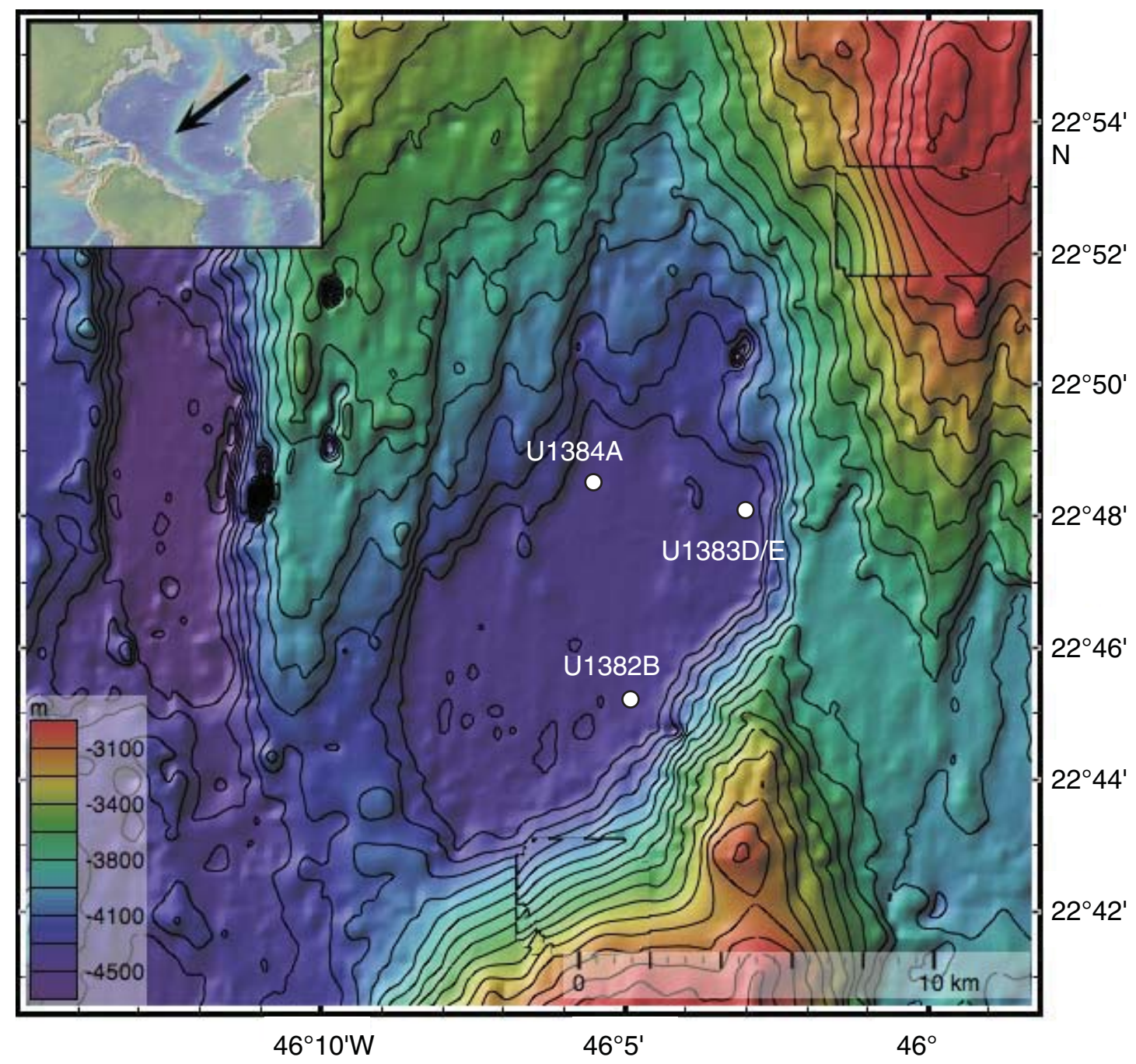


Figure F2. SEDEX sequential extraction results for (A) Hole U1384A, located on the northern edge, and (B) Hole U1382B, located on the southern edge of the sediment pond. The concentrations of each solid-phase P reservoir are expressed in units of $\mu \mathrm{mol}$ of P per gram of sediment ground dry weight (gdw) as a function of depth. Basement depths $=94.71$ mbsf in Hole U1384A and 90 mbsf in Hole U1382B (Expedition 336 Scientists, 2012).

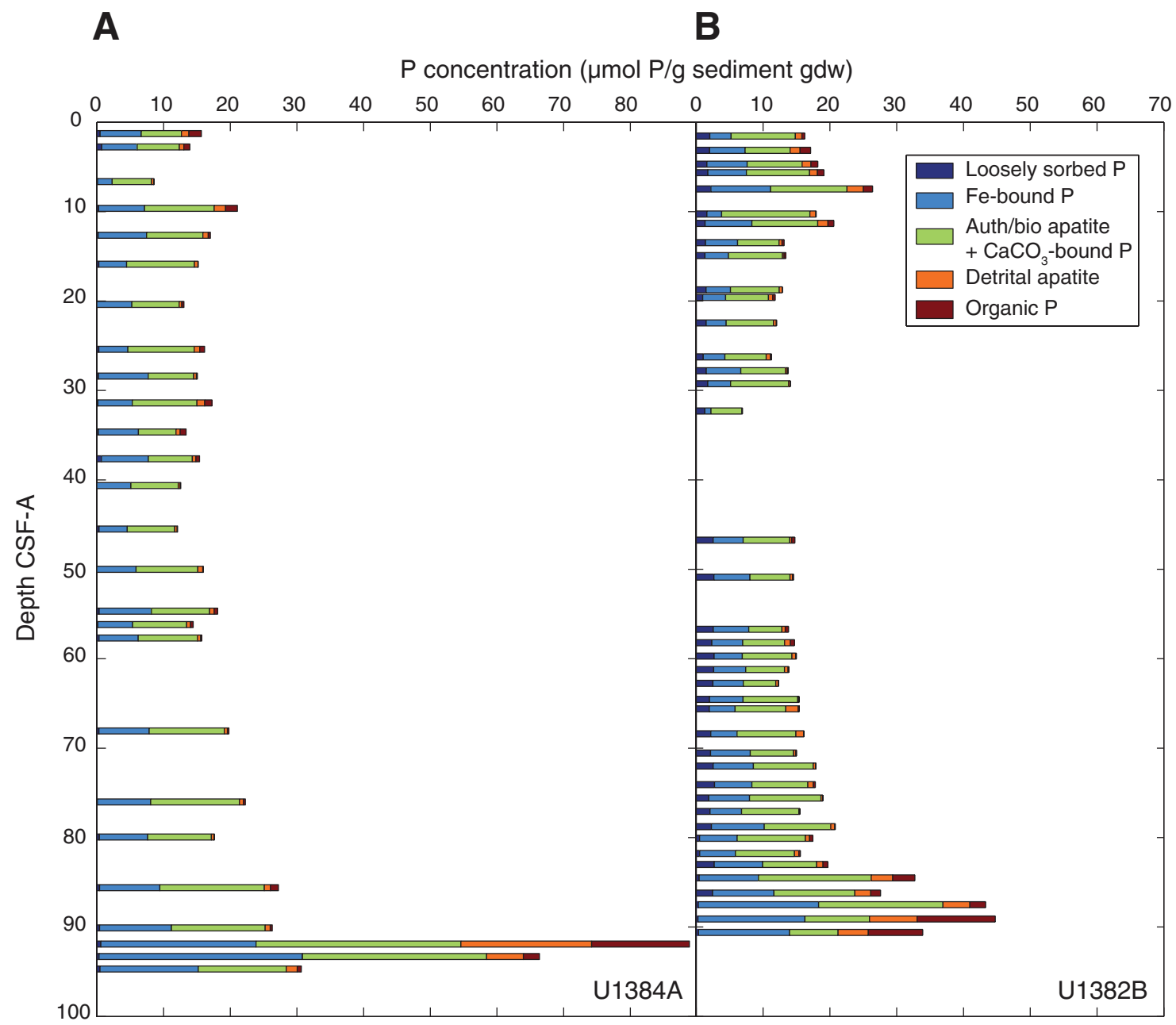


Figure F3. SEDEX sequential extraction results for (A) Holes U1383D and (B) U1383E, both located on the eastern edge of the sediment pond. Concentrations of each solid-phase P reservoir are expressed in units of $\mu \mathrm{mol}$ of P per gram of sediment ground dry weight ( $\mathrm{gdw})$ as a function of depth. Basement depths $=43.3 \mathrm{mbsf}$ in Hole U1383D and 43.21 mbsf in Hole U1383E (Expedition 336 Scientists, 2012).

A

B

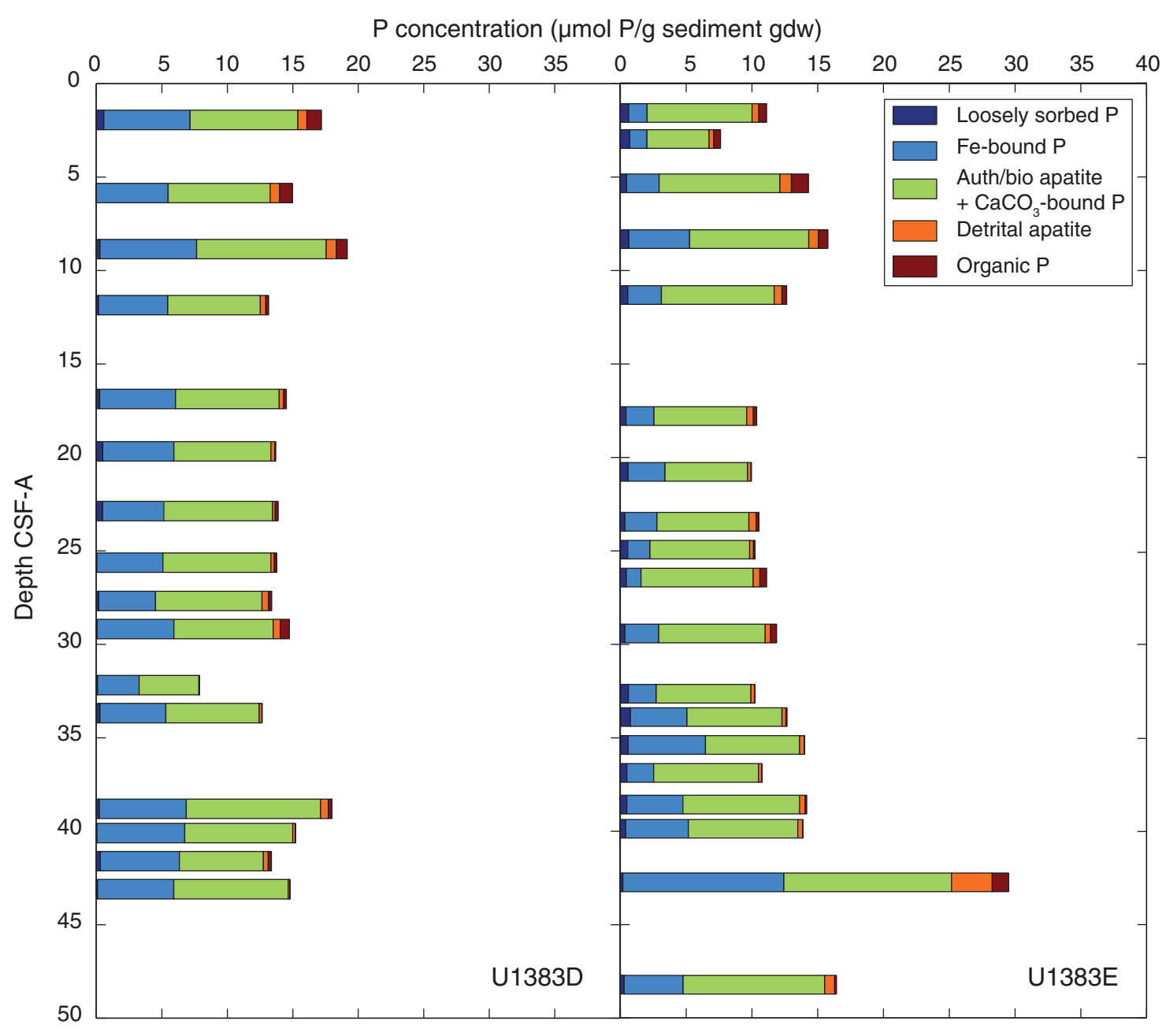


Table T1. Solid-phase P concentration data, Hole U1384A.

\begin{tabular}{|c|c|c|c|c|c|c|}
\hline \multirow[b]{2}{*}{$\begin{array}{l}\text { Core, } \\
\text { section }\end{array}$} & \multirow{2}{*}{$\begin{array}{l}\text { Average } \\
\text { depth } \\
\text { (mbsf) }\end{array}$} & \multicolumn{5}{|c|}{$\mathrm{P}(\mu \mathrm{mol} / \mathrm{g}$ sediment $[\mathrm{gdw}])$} \\
\hline & & Loosely sorbed & Iron-bound & $\begin{array}{l}\text { Auth/bio apatite }+ \\
\mathrm{CaCO}_{3} \text {-bound }\end{array}$ & $\begin{array}{l}\text { Detrital } \\
\text { apatite }\end{array}$ & Organic \\
\hline \multicolumn{7}{|c|}{ 336-U1384A- } \\
\hline $1 \mathrm{H}-1$ & 1.27 & 0.48 & 6.16 & 6.03 & 1.13 & 1.87 \\
\hline $1 \mathrm{H}-2$ & 2.77 & 0.71 & 5.33 & 6.31 & 0.68 & 0.91 \\
\hline $2 \mathrm{H}-2$ & 6.64 & 0.08 & 2.20 & 5.88 & 0.37 & 0.00 \\
\hline $2 \mathrm{H}-4$ & 9.64 & 0.26 & 6.88 & 10.45 & 1.71 & 1.77 \\
\hline $2 \mathrm{H}-6$ & 12.64 & 0.18 & 7.27 & 8.43 & 0.82 & 0.32 \\
\hline $3 \mathrm{H}-2$ & 15.88 & 0.28 & 4.17 & 10.17 & 0.54 & 0.00 \\
\hline $3 \mathrm{H}-5$ & 20.38 & 0.00 & 5.24 & 7.08 & 0.39 & 0.33 \\
\hline $4 \mathrm{H}-2$ & 25.39 & 0.29 & 4.34 & 9.95 & 0.84 & 0.73 \\
\hline $4 \mathrm{H}-4$ & 28.39 & 0.21 & 7.48 & 6.81 & 0.42 & 0.14 \\
\hline $4 \mathrm{H}-6$ & 31.39 & 0.13 & 5.17 & 9.70 & 1.16 & 1.09 \\
\hline $5 \mathrm{H}-2$ & 34.64 & 0.19 & 6.03 & 5.62 & 0.63 & 0.90 \\
\hline $5 \mathrm{H}-4$ & 37.64 & 0.67 & 7.04 & 6.60 & 0.52 & 0.55 \\
\hline $5 \mathrm{H}-6$ & 40.64 & 0.00 & 5.10 & 7.09 & 0.29 & 0.10 \\
\hline $6 \mathrm{H}-2$ & 45.50 & 0.31 & 4.23 & 7.12 & 0.37 & 0.06 \\
\hline $6 \mathrm{H}-5$ & 50.00 & 0.00 & 5.87 & 9.27 & 0.74 & 0.08 \\
\hline $7 \mathrm{H}-2$ & 54.68 & 0.33 & 7.84 & 8.70 & 0.71 & 0.51 \\
\hline $7 \mathrm{H}-3$ & 56.18 & 0.12 & 5.23 & 8.10 & 0.59 & 0.39 \\
\hline $7 \mathrm{H}-4$ & 57.68 & 0.32 & 5.86 & 8.90 & 0.48 & 0.14 \\
\hline $8 \mathrm{H}-5$ & 68.07 & 0.30 & 7.53 & 11.27 & 0.46 & 0.23 \\
\hline $9 \mathrm{H}-4$ & 76.01 & 0.06 & 7.99 & 13.31 & 0.58 & 0.31 \\
\hline $9 \mathrm{H}-7$ & 79.93 & 0.37 & 7.24 & 9.57 & 0.38 & 0.06 \\
\hline $10 \mathrm{H}-4$ & 85.60 & 0.40 & 9.03 & 15.67 & 0.91 & 1.20 \\
\hline $10 \mathrm{H}-7$ & 90.10 & 0.40 & 10.74 & 14.08 & 0.77 & 0.30 \\
\hline $11 \mathrm{H}-2$ & 91.90 & 0.59 & 23.27 & 30.73 & 19.59 & 14.67 \\
\hline $11 \mathrm{H}-3$ & 93.20 & 0.12 & 29.40 & 32.05 & 8.10 & 3.33 \\
\hline $11 \mathrm{H}-3$ & 93.40 & 0.50 & 31.57 & 23.27 & 2.94 & 1.48 \\
\hline $11 \mathrm{H}-4$ & 94.60 & 0.58 & 15.51 & 12.73 & 1.93 & 0.79 \\
\hline $11 \mathrm{H}-4$ & 94.80 & 0.33 & 13.96 & 13.66 & 1.39 & 0.43 \\
\hline
\end{tabular}

gdw = ground dry weight. 
Table T2. Solid-phase P concentration data, Hole U1382B.

\begin{tabular}{|c|c|c|c|c|c|c|}
\hline \multirow[b]{2}{*}{$\begin{array}{l}\text { Core, } \\
\text { section }\end{array}$} & \multirow{2}{*}{$\begin{array}{l}\text { Average } \\
\text { depth } \\
\text { (mbsf) }\end{array}$} & \multicolumn{5}{|c|}{$\mathrm{P}(\mu \mathrm{mol} / \mathrm{g}$ sediment $[\mathrm{gdw}])$} \\
\hline & & Loosely sorbed & Iron-bound & $\begin{array}{l}\text { Auth/bio apatite }+ \\
\mathrm{CaCO}_{3} \text {-bound }\end{array}$ & $\begin{array}{l}\text { Detrital } \\
\text { apatite }\end{array}$ & Organic \\
\hline \multicolumn{7}{|c|}{ 336-U1382B- } \\
\hline $1 \mathrm{H}-2$ & 1.55 & 2.03 & 3.18 & 9.62 & 0.98 & 0.44 \\
\hline $1 \mathrm{H}-3$ & 3.15 & 1.99 & 5.31 & 6.74 & 1.50 & 1.57 \\
\hline $1 \mathrm{H}-4$ & 4.70 & 1.58 & 6.03 & 8.24 & 1.32 & 1.03 \\
\hline $1 \mathrm{H}-5$ & 5.65 & 1.73 & 5.77 & 9.45 & 1.18 & 0.98 \\
\hline $2 \mathrm{H}-2$ & 7.47 & 2.18 & 8.94 & 11.43 & 2.45 & 1.39 \\
\hline $2 \mathrm{H}-4$ & 10.27 & 1.59 & 2.19 & 13.21 & 0.88 & 0.07 \\
\hline $2 \mathrm{H}-5$ & 11.32 & 1.31 & 7.01 & 9.87 & 1.52 & 0.89 \\
\hline $2 \mathrm{H}-6$ & 13.47 & 1.38 & 4.80 & 6.20 & 0.44 & 0.33 \\
\hline $2 \mathrm{H}-7$ & 14.92 & 1.30 & 3.52 & 8.08 & 0.25 & 0.27 \\
\hline $3 \mathrm{H}-3$ & 18.75 & 1.46 & 3.66 & 7.30 & 0.44 & 0.00 \\
\hline $3 \mathrm{H}-4$ & 19.60 & 0.95 & 3.43 & 6.41 & 0.65 & 0.37 \\
\hline $3 \mathrm{H}-5$ & 22.45 & 1.47 & 3.00 & 7.07 & 0.43 & 0.07 \\
\hline $4 \mathrm{H}-2$ & 26.25 & 1.04 & 3.23 & 6.19 & 0.63 & 0.18 \\
\hline $4 \mathrm{H}-3$ & 27.80 & 1.50 & 5.16 & 6.67 & 0.27 & 0.17 \\
\hline $4 \mathrm{H}-4$ & 29.25 & 1.71 & 3.41 & 8.69 & 0.24 & 0.00 \\
\hline $4 \mathrm{H}-6$ & 32.30 & 1.27 & 0.93 & 4.61 & 0.11 & 0.00 \\
\hline $6 \mathrm{H}-3$ & 46.74 & 2.52 & 4.50 & 6.97 & 0.30 & 0.47 \\
\hline $6 \mathrm{H}-7$ & 50.88 & 2.63 & 5.40 & 5.99 & 0.36 & 0.18 \\
\hline $7 \mathrm{H}-3$ & 56.71 & 2.54 & 5.31 & 4.96 & 0.51 & 0.49 \\
\hline $7 \mathrm{H}-4$ & 58.21 & 2.33 & 4.62 & 6.26 & 0.88 & 0.61 \\
\hline $7 \mathrm{H}-5$ & 59.71 & 2.66 & 4.22 & 7.43 & 0.57 & 0.11 \\
\hline $7 \mathrm{H}-6$ & 61.21 & 2.59 & 4.81 & 5.80 & 0.53 & 0.14 \\
\hline $7 \mathrm{H}-7$ & 62.76 & 2.49 & 4.55 & 4.85 & 0.40 & 0.03 \\
\hline $8 \mathrm{H}-2$ & 64.55 & 1.98 & 5.02 & 8.19 & 0.19 & 0.00 \\
\hline $8 \mathrm{H}-3$ & 65.60 & 1.95 & 3.86 & 7.59 & 1.83 & 0.19 \\
\hline $8 \mathrm{H}-5$ & 68.40 & 2.17 & 3.92 & 8.81 & 1.21 & 0.02 \\
\hline $8 \mathrm{H}-6$ & 70.55 & 2.14 & 5.93 & 6.48 & 0.34 & 0.13 \\
\hline $8 \mathrm{H}-7$ & 72.00 & 2.52 & 6.03 & 8.95 & 0.41 & 0.00 \\
\hline $9 \mathrm{H}-2$ & 74.08 & 2.72 & 5.60 & 8.37 & 0.83 & 0.30 \\
\hline $9 \mathrm{H}-3$ & 75.58 & 1.85 & 6.12 & 10.69 & 0.29 & 0.00 \\
\hline $9 \mathrm{H}-4$ & 77.08 & 2.06 & 4.72 & 8.58 & 0.14 & 0.00 \\
\hline $9 \mathrm{H}-5$ & 78.78 & 2.27 & 7.90 & 9.96 & 0.60 & 0.09 \\
\hline $9 \mathrm{H}-6$ & 80.08 & 0.49 & 5.62 & 10.21 & 0.63 & 0.51 \\
\hline $9 \mathrm{H}-7$ & 81.78 & 0.50 & 5.35 & 8.85 & 0.64 & 0.24 \\
\hline $10 \mathrm{H}-2$ & 83.01 & 2.68 & 7.26 & 8.09 & 0.95 & 0.71 \\
\hline $10 \mathrm{H}-3$ & 84.51 & 0.43 & 8.87 & 16.89 & 3.23 & 3.31 \\
\hline $10 \mathrm{H}-4$ & 86.21 & 2.45 & 9.17 & 12.12 & 2.38 & 1.47 \\
\hline $10 \mathrm{H}-5$ & 87.51 & 0.30 & 18.00 & 18.63 & 4.02 & 2.37 \\
\hline $10 \mathrm{H}-6$ & 89.01 & 0.33 & 20.25 & 2.73 & 3.42 & 16.98 \\
\hline $10 \mathrm{H}-6$ & 89.21 & 0.17 & 11.80 & 16.60 & 10.82 & 6.43 \\
\hline $10 \mathrm{H}-7$ & 90.51 & 0.50 & 16.94 & 8.28 & 5.68 & 12.98 \\
\hline $10 \mathrm{H}-7$ & 90.71 & 0.17 & 10.31 & 6.27 & 3.36 & 3.32 \\
\hline
\end{tabular}

gdw = ground dry weight. 
Table T3. Solid-phase P concentration data, Holes U1383D-U1383E.

\begin{tabular}{|c|c|c|c|c|c|c|}
\hline \multirow[b]{2}{*}{$\begin{array}{l}\text { Core, } \\
\text { section }\end{array}$} & \multirow{2}{*}{$\begin{array}{l}\text { Average } \\
\text { depth } \\
\text { (mbsf) }\end{array}$} & \multicolumn{5}{|c|}{$\mathrm{P}(\mu \mathrm{mol} / \mathrm{g}$ sediment $[\mathrm{gdw}])$} \\
\hline & & Loosely sorbed & Iron-bound & $\begin{array}{l}\text { Auth/bio apatite }+ \\
\mathrm{CaCO}_{3} \text {-bound }\end{array}$ & $\begin{array}{l}\text { Detrital } \\
\text { apatite }\end{array}$ & Organic \\
\hline \multicolumn{7}{|c|}{ 336-U1383D- } \\
\hline $1 \mathrm{H}-2$ & 1.94 & 0.56 & 6.59 & 8.23 & 0.69 & 1.12 \\
\hline $2 \mathrm{H}-1$ & 5.85 & 0.00 & 5.48 & 7.79 & 0.73 & 0.97 \\
\hline $2 \mathrm{H}-3$ & 8.85 & 0.27 & 7.38 & 9.89 & 0.78 & 0.82 \\
\hline $2 \mathrm{H}-5$ & 11.85 & 0.16 & 5.28 & 7.07 & 0.42 & 0.21 \\
\hline $3 \mathrm{H}-2$ & 16.87 & 0.24 & 5.80 & 7.91 & 0.34 & 0.21 \\
\hline $3 \mathrm{H}-4$ & 19.67 & 0.48 & 5.43 & 7.41 & 0.30 & 0.07 \\
\hline $3 \mathrm{H}-6$ & 22.87 & 0.47 & 4.67 & 8.30 & 0.21 & 0.22 \\
\hline $4 \mathrm{H}-2$ & 25.63 & 0.03 & 5.04 & 8.25 & 0.25 & 0.18 \\
\hline $4 \mathrm{H}-3$ & 27.68 & 0.18 & 4.34 & 8.13 & 0.50 & 0.25 \\
\hline $4 \mathrm{H}-4$ & 29.18 & 0.05 & 5.86 & 7.59 & 0.54 & 0.70 \\
\hline $4 \mathrm{H}-6$ & 32.18 & 0.08 & 3.18 & 4.56 & 0.03 & 0.00 \\
\hline $4 \mathrm{H}-7$ & 33.68 & 0.27 & 5.03 & 7.13 & 0.21 & 0.00 \\
\hline $5 \mathrm{H}-4$ & 38.80 & 0.22 & 6.64 & 10.27 & 0.59 & 0.28 \\
\hline $5 \mathrm{H}-5$ & 40.10 & 0.05 & 6.69 & 8.26 & 0.20 & 0.00 \\
\hline $5 \mathrm{H}-6$ & 41.60 & 0.30 & 6.03 & 6.42 & 0.37 & 0.25 \\
\hline $5 \mathrm{H}-7$ & 43.10 & 0.08 & 5.82 & 8.74 & 0.13 & 0.00 \\
\hline \multicolumn{7}{|c|}{ 336-U1383E- } \\
\hline $1 \mathrm{H}-1$ & 1.40 & 0.62 & 1.41 & 7.99 & 0.51 & 0.59 \\
\hline $1 \mathrm{H}-2$ & 2.80 & 0.70 & 1.32 & 4.72 & 0.36 & 0.52 \\
\hline $2 \mathrm{H}-2$ & 5.18 & 0.47 & 2.47 & 9.18 & 0.88 & 1.30 \\
\hline $2 \mathrm{H}-4$ & 8.18 & 0.64 & 4.63 & 9.05 & 0.73 & 0.72 \\
\hline $2 \mathrm{H}-6$ & 11.18 & 0.56 & 2.56 & 8.57 & 0.59 & 0.36 \\
\hline $3 \mathrm{H}-4$ & 17.68 & 0.44 & 2.12 & 7.05 & 0.50 & 0.26 \\
\hline $3 \mathrm{H}-6$ & 20.68 & 0.59 & 2.80 & 6.27 & 0.27 & 0.03 \\
\hline $4 \mathrm{H}-2$ & 23.36 & 0.35 & 2.43 & 6.99 & 0.55 & 0.21 \\
\hline $4 \mathrm{H}-3$ & 24.86 & 0.55 & 1.69 & 7.58 & 0.29 & 0.11 \\
\hline $4 \mathrm{H}-4$ & 26.36 & 0.45 & 1.12 & 8.52 & 0.51 & 0.51 \\
\hline $4 \mathrm{H}-6$ & 29.36 & 0.35 & 2.57 & 8.09 & 0.41 & 0.45 \\
\hline $5 \mathrm{H}-1$ & 32.60 & 0.60 & 2.12 & 7.20 & 0.30 & 0.00 \\
\hline $5 \mathrm{H}-2$ & 33.85 & 0.76 & 4.30 & 7.22 & 0.30 & 0.09 \\
\hline $5 \mathrm{H}-3$ & 35.35 & 0.59 & 5.88 & 7.15 & 0.38 & 0.00 \\
\hline $5 \mathrm{H}-4$ & 36.85 & 0.49 & 2.04 & 7.97 & 0.26 & 0.01 \\
\hline $5 \mathrm{H}-5$ & 38.55 & 0.49 & 4.26 & 8.88 & 0.42 & 0.12 \\
\hline $5 \mathrm{H}-6$ & 39.85 & 0.40 & 4.76 & 8.32 & 0.39 & 0.00 \\
\hline $6 \mathrm{H}-2$ & 42.63 & 0.24 & 13.77 & 13.62 & 3.58 & 1.00 \\
\hline $6 \mathrm{H}-2$ & 42.83 & 0.13 & 10.72 & 11.85 & 2.62 & 1.50 \\
\hline $6 \mathrm{H}-6$ & 48.23 & 0.29 & 4.48 & 10.76 & 0.76 & 0.13 \\
\hline
\end{tabular}

gdw = ground dry weight. 